\title{
The population genetic consequences of diapause in Eudiaptomus copepods
}

\author{
Andrew J. Bohonak ${ }^{1}$, Matthew D. Holland ${ }^{2,3}$, Barbara Santer ${ }^{4}$, \\ Martina Zeller ${ }^{4}$, Colleen M. Kearns ${ }^{2}$ and Nelson G. Hairston, Jr. ${ }^{2}$
}

With 5 figures and 2 tables

\begin{abstract}
Prolonged diapause (extended dormancy) is thought to greatly influence evolution in freshwater invertebrates by lengthening generation time, promoting higher levels of dispersal among populations by wind or animal vectors, and increasing effective population size. However, empirical tests of these predictions are relatively rare. Comparative studies can be informative in this regard, if the comparisons involve sympatric, closely related species that differ only in the presence or absence of a dormant life history stage. We built upon a previous study by ZELLER et al. (2006), which used this approach to study patterns of microsatellite variation in Eudiaptomus copepods from northern Europe. E. graciloides possesses diapausing eggs and adults, whereas the closely related species $E$. gracilis is ecologically and trophically similar but lacks diapause. To separate further historical processes from recent anthropogenic influences, we examined mitochondrial DNA sequence variation in these species from three lakes in northern Germany where they are sympatric. Biotic and abiotic variation among contemporary and historical populations was minimized by focusing on hydrologically linked lakes separated by less than $10 \mathrm{~km}$.

E. gracilis and E. graciloides possess very different patterns of mtDNA variation. Both species possess significant population structure on small spatial scales, but $E$. graciloides has far fewer alleles despite a larger average divergence. Phylogeographic analyses and Bayesian skyline plots revealed evidence for historic population expansions in both species, with the growth phase beginning thousands of generations earlier in E. gracilis than in E. graciloides. Levels of genetic diversity suggest that effective population size may be an order of magnitude larger in E. gracilis than in E. graciloi-
\end{abstract}

1 Authors' addresses: San Diego State University, Department of Biology, 5500 Campanile Drive, San Diego, CA 92182-4614, USA.

2 Cornell University, Department of Ecology and Evolutionary Biology, Corson Hall, Ithaca, NY 14853-2701, USA.

3 Current address: Center for Population Biology, University of California, One Shields Avenue, Davis, CA 95616, USA.

4 Max-Planck-Institute for Limnology, Postfach 165, D-24302 Plön, Germany.

* Corresponding author; E-mail: bohonak@sciences.sdsu.edu 
des, even though E. graciloides possesses a diapausing egg bank. Although this would seem to be an ideal system for quantifying the genetic role of diapause, we found no support for the assumption that freshwater invertebrates with diapause categorically possess larger effective population sizes and higher rates of gene flow than closely related species that lack diapause.

Key words: effective population size, Eudiaptomus gracilis, Eudiaptomus graciloides, genetic population structure, mitochondrial DNA.

\section{Introduction}

Prolonged diapause (i.e., lasting longer than a single active generation) is generally assumed to have pronounced effects on patterns of genetic variation in freshwater invertebrates. "Banks" of diapausing eggs typically contain billions of propagules that can be viable for several decades (e. g., Hairston et al. 1995, Hairston 1996, Cousyn et al. 2001), increasing effective population size beyond individuals found in the water column, and reducing the relative importance of genetic drift. Extended diapause can also substantially increase generation times, slowing the rates of microevolutionary processes (HAIRSTON \& De Stasio 1988). For traits that experience fluctuating selection, extended diapause can in theory act to maintain genetic variation (ELLNER \& HAIRSTON 1994, HEDRICK 1995), which has been shown for a population of diaptomid copepods (ELLNER et al. 1999). Diapause is also believed to promote higher levels of dispersal among populations, since many encysted diapausing eggs are resistant to harsh environmental conditions and possess attributes that appear to promote transport by vertebrates or wind (e. g., BRENDONCK et al. 1992, Korovchinsky \& BoIKova 1996, BRENDONCK \& RidDoch 1999).

The estimation of movement among populations (dispersal and gene flow) occupies a particularly important role in population biology, community ecology and conservation. Theoretical debates about topics as varied as population persistence, local adaptation and community assembly rules are based in part on different underlying assumptions about the spatial and temporal frequency of movement (e. g., Belyea \& LanCaster 1999, Harrison \& BrUna 1999, KAWECKI \& EBERT 2004). There is no general consensus regarding the extent to which populations of freshwater invertebrates are connected by dispersal and gene flow, and it is not even clear that there should be a consensus. Most species of zooplankton and other freshwater invertebrates have explicitly or implicitly been assumed to disperse widely and often through various means (reviewed by Bilton et al. 2001, BohonaK \& Jenkins 2003). However, experiments specifically focused on estimating dispersal have found that this generalization does not hold, since the range of dispersal rates runs from very low to very high for any particular spatial scale (e.g., JENKINS \& UNDERWOOD 
1998, Brendonck \& Riddoch 1999, Cáceres \& Soluk 2002, Cohen \& Shurin 2003, Louette \& De Meester 2005). In numerous studies of zooplankton, the highest gene flow estimates are not always found in species with attributes that are thought to confer high dispersal ability (BoILEAU et al. 1992). In the most striking cases, significant genetic differences have been found at even the smallest spatial scales (e.g., WEIDER \& Hebert 1987, VANoVerbeke \& De Meester 1997, Gómez et al. 2002).

For neutral genetic markers, genetic differences among populations are primarily due to a balance between mutation, drift and gene flow. Interpreting empirical data is not straightforward, because it is almost always the case that observed patterns can be explained by more than one parameter combination. The variety of interpretations increases even further under nonequilibrium conditions, because the genetic signature of a founder event (or historically higher rates of gene flow) may last for thousands of generations or longer (Boileau et al. 1992, BohonaK \& Roderick 2001, De Meester et al. 2002). As a result, freshwater biologists have interpreted levels of genetic divergence on a case-by-case basis by invoking specific combinations of founder effects, drift, dispersal, gene flow and the uncoupling of dispersal and gene flow due to local adaptation. For example, the "monopolization hypothesis" (DE MEEsTER et al. 2002) assumes that zooplankton dispersal rates are relatively high, and that diapausing egg banks exacerbate genetic founder effects by inflating population sizes and lengthening generation times. However, BOHONAK \& JENKINS (2003) speculated that the majority of freshwater invertebrates may not possess the unique combination of parameters (e.g., dispersal rate, generation time) required for this hypothesis.

Comparisons between organisms with different life histories provide one way to assess the assumed genetic consequences of prolonged diapause. The ideal contrast would be between sympatric, closely related species that differ only in the presence or absence of a dormant life history stage. ZELLER et al. (2006) used this approach to study patterns of microsatellite variation in $\mathrm{Eu}$ diaptomus copepods from northern Europe at different spatial scales. E. graciloides, which is known to produce diapausing eggs and adults, shows patterns of isolation by distance (IBD) on small and very large spatial scales $(0-100 \mathrm{~km}$ and up to $1300 \mathrm{~km}$ ) but not intermediate scales $(100-1000 \mathrm{~km})$. Patterns of IBD (decreasing genetic similarity with increasing geographic distance) generally indicate that gene flow is too infrequent and/or spatially restricted to create panmixia over the spatial scale being studied (SLATKIN 1993). The closely related species $E$. gracilis is ecologically similar, regionally sympatric, and often coexists with E. graciloides in the same lake (KIEFER 1978, HoFmanN 1979, NAUWERCK 1980, Adrian 1997). However, E. gracilis does not produce diapausing eggs (SANTER et al. 2000), and does not display IBD on small or intermediate scales (ZELLER et al. 2006). (Populations $>1000 \mathrm{~km}$ were not stud- 
ied for this species). High variance in the E. gracilis IBD plot on small scales could reflect recent, irregular colonization patterns and high levels of drift (HutCHINSON \& TEMPLETON 1999). However, persistent founder effects are actually expected to be weaker in E. gracilis than in E. graciloides, because diapausing egg banks in $E$. graciloides should greatly increase population size and slow the approach to genetic equilibrium. ZELLER et al. (2006) hypothesized that small-scale dispersal rates are higher in E. graciloides than in $E$. gracilis, eliminating founder effects in the former but not the latter. Alternative interpretations for all three spatial scales may involve specific dispersal vectors or historical processes.

To separate further the effects of population history, drift and gene flow in these two species, we examined mitochondrial DNA sequence variation in three lakes from northern Germany where they are sympatric. The use of DNA sequence variation offers some advantages over microsatellite markers, including a wider array of analyses to assess historical demographic changes. Two of the three lakes are connected by a river system that has been modified for boat traffic, facilitating the separation of very recent anthropologic changes in the landscape from those that took place during the post-Pleistocene. Our overall goals were to build upon the results of ZeLLER et al. (2006) and assess the role of diapause in shaping genetic population structure. We specifically focused on whether prolonged dormancy in E. graciloides is associated with more persistent founder effects, increased rates of gene flow, increased effective population sizes, and less dramatic bottlenecks in population size when compared to E. gracilis.

\section{Material and methods}

Eudiaptomus gracilis and E. graciloides were collected from the open water of three lakes in Schleswig-Holstein, Germany: the Grosser Plöner See, the Keller See and the Schöhsee. Collections were taken during May 1999 (approximately 5 individuals per species per lake) and June and July 2000 (ca. 15 individuals per species per lake). The Keller See is approximately $8.6 \mathrm{~km}$ east and upstream of the Grosser Plöner See, and the two lakes are separated by the small river Schwentine, and two intervening lakes (the Dieksee and the Behler See successively; see Fig. 5). The outflow from the Schöhsee enters the Behler See through a small stream inhospitable to planktonic copepods that flows only when the water level in Schöhsee is very high. The Behler See then flows into the Grosser Plöner See, with a total hydrologic distance between the Schöhsee and the Grosser Plöner See of ca. $2.3 \mathrm{~km}$. These three lakes were also sampled by ZELLER et al. (2006), although this study used different individuals.

Adult females were sorted, identified and frozen at $-70{ }^{\circ} \mathrm{C}$ prior to DNA extraction with Chelex-100 beads (Biorad, WALsH et al. 1991). A portion of the mitochondrial gene cytochrome oxidase I (CO I) was amplified using the primer pair C1-J-1718 and 
C1-N-2191 (Simon et al. 1994). $50 \mu$ reactions contained $2 \mu$ of $1: 10$ diluted DNA template, $1.5 \mathrm{mM} \mathrm{MgCl}_{2}, 200 \mu \mathrm{M}$ of each DNTP, $400 \mathrm{nM}$ each primer, 1 unit each Taq polymerase and Platinum Taq antibody (GibcoBRL) and reaction buffer. Samples were amplified with a 2 minute hot start at $94{ }^{\circ} \mathrm{C}$ followed by 35 cycles of $94{ }^{\circ} \mathrm{C}(30 \mathrm{sec}$.), $52{ }^{\circ} \mathrm{C}\left(30 \mathrm{sec}\right.$.), $72{ }^{\circ} \mathrm{C}\left(45 \mathrm{sec}\right.$.) and a final 7 minute extension at $72{ }^{\circ} \mathrm{C}$. PCR products were verified on a $1.5 \%$ agarose gel with ethidium bromide staining and purified using Qiaquick kits (Qiagen). Following cycle sequencing with BigDye reaction mix (Perkin Elmer), sequences were visualized on an ABI 377 automated sequencer. In approximately half of the individuals, PCR products were sequenced in both directions to clarify ambiguous sites. After excluding beginning sites that could not be reliably scored in all individuals, sequence length was $437 \mathrm{bp}$. Because there were no insertions or deletions, sequences were aligned visually using Sequencher v. 4.0 (Gene Codes Corporation). Sequences have been submitted to NCBI GenBank (accession numbers DQ457129DQ457160 and DQ459087-DQ459093). The analyses described below were conducted using all individuals (whether or not they possessed identical haplotypes).

Variation for each species (over all individuals, and within each lake) was summarized using the standard statistics $K$ (number of alleles = unique haplotypes), $S$ (number of segregating sites), and $\pi$ (nucleotide diversity: the average pairwise divergence between all individuals). We estimated the population genetic parameter $\theta=4 N_{e} \mu$ from $K, S$ and $\pi$. (This parameter determines the amount of diversity in a population and the shape of a gene genealogy, where $N_{e}$ is the effective population size and $\mu$ is the mutation rate: HaLliburTon 2004). Divergence among lakes was tested for statistical significance using exact tests (100,000 Markov chain steps), and quantified using Wright's (1931) $F_{S T}$ and ExCOFFIER et al.'s (1992) $\Phi_{S T}(10,000$ randomizations or permutations in each case). In contrast to $F_{S T}, \Phi_{S T}$ considers the genealogical or evolutionary distance among alleles, in addition to their frequencies in each population. Because the distances among alleles were small, we used the matrix of observed pairwise distances for these calculations, rather than a corrected distance measure. We also compared patterns of mtDNA variation from this study with the microsatellite data of ZELLER et al. (2006). For each species, we calculated expected heterozygosity $H_{e}$ in each lake for microsatellites, and we calculated differentiation among lakes as $F_{S T}$. Statistical significance for microsatellite $F_{S T}$ in $E$. gracilis was estimated by bootstrapping over seven variable loci. Significance for E. graciloides could not be determined because only three loci were available. Calculations were conducted in Arlequin (SCHNeIDER et al. 2001), Genepop on the Web (RAYMond \& Rousset 1995) and Fstat (GoudeT 1995).

We conducted two "neutrality tests" for each species (overall, and within each lake) that compare the above summary statistics to expectations from neutral gene genealogies at equilibrium: TAJIMA's (1989) $D$ (which compares $\theta_{\mathrm{S}}$ to $\theta_{\pi}$ ), and FU's (1997) $F$ (which evaluates $K$, conditional on $\pi$ ). Statistical significance was evaluated based on 10,000 simulated samples for $D$ and $F$ under the null hypothesis of neutral evolution in a stable population. For each species, we also compared the mismatch distribution shape to that expected under an expanding population using 10,000 bootstrapped replicates. (The mismatch distribution is a histogram of pairwise genetic distances between all samples, and its shape can reflect recent demographic changes: Ro- 
GERS \& HARPENDING 1992). The null hypothesis for this test (as implemented in Arlequin) is population expansion.

We estimated the genealogical relationships within each species using two methods. First, we used the parsimony-based network reconstruction method of TEMPLEtON and colleagues (e.g., Templeton et al. 1992, Templeton 1998) because it explicitly allows for multifurcations and the persistence of ancestral nodes. Thus, it is likely to be more appropriate for recently diverged alleles than standard phylogenetic tree-building techniques (reviewed by Posada \& Crandall 2001). Mitochondrial haplotype networks were estimated for each species using TCS v. 1.13 (CLEMENT et al. 2000) and nested cladistic relationships were inferred using the algorithms of TEMPLETON et al. (1987, 1992). Non-random geographic associations among haplotypes were tested statistically using 10,000 permutations for each clade with the computer program GeoDis (PosadA et al. 2000), and the results were interpreted using the most recent inference key (2005) of TEMPLETON and collaborators. The dichotomous inference key examines the geographic distribution of each clade member, assessing whether patterns are consistent with processes such as restricted gene flow (i.e., isolation by distance), allopatric fragmentation or long-distance colonization. TEMPLETON $(1998,2004)$ provides an overview of the entire process, known as nested clade phylogenetic analysis (NCPA). Some critics have argued that the final phase of NCPA (the inference key) does not appropriately distinguish among alternative historical hypotheses (KNOwLES \& MAdDison 2002), although Templeton (2004) has addressed many of those criticisms and made some modifications to the inference key. For this study, we treated the inferences from NCPA as working hypotheses, and evaluated them based on the neutrality tests described above and qualitative patterns in the gene genealogies.

We also estimated the genealogy for each species with a Bayesian analysis conducted in BEAST (Drummond et al. 2002, Drummond \& Rambaut 2003). To help interpret the results of the neutrality tests and NCPA, we used BEAST to estimate $N_{e} \mu$ backwards in time based on coalescent events in the genealogy. The results are presented as a Bayesian "skyline plot" generated in TRACER v. 1.2.1 (Drummond et al. 2005). Assuming that mutation rates are equal in E. gracilis and E. graciloides, the skyline plot depicts relative estimates of population sizes throughout the species' histories. To interpret the historical processes represented in these plots, we estimated generation times for each species using published and unpublished data.

\section{Results}

E. gracilis and E. graciloides possessed remarkably different patterns of nucleotide-level variation even though all mtDNA substitutions within each species were silent (i.e., they do not change amino acid composition and are therefore selectively neutral). The maximum divergence was $1.8 \%$ within $E$. gracilis, and $3.0 \%$ within the diapausing species E. graciloides. Consequently, the average sequence divergence $\pi$ was higher in E. graciloides ( 0.0087 vs. 0.0075 in E. gracilis). However, E. gracilis had twice as many polymorphic 
Table 1. Summary of mtDNA variation within lakes and across all three lakes, in terms of sample size $N$, unique alleles $K$, estimates of $\theta$, and two neutrality tests. * $\mathrm{p} \leq 0.05$; for $F, \mathrm{p}<0.05$ corresponds to a $2 \%$ cutoff in the randomized distribution, rather than $5 \%$ (Fu 1997).

\begin{tabular}{|c|c|c|c|c|c|c|c|c|}
\hline & \multicolumn{4}{|c|}{ E. gracilis } & \multicolumn{4}{|c|}{ E. graciloides } \\
\hline & $\begin{array}{l}\text { All } \\
\text { individuals }\end{array}$ & $\begin{array}{l}\text { Grosser } \\
\text { Plöner See }\end{array}$ & $\begin{array}{l}\text { Keller } \\
\text { See }\end{array}$ & Schöhsee & $\begin{array}{l}\text { All } \\
\text { individuals }\end{array}$ & $\begin{array}{l}\text { Grosser } \\
\text { Plöner See }\end{array}$ & $\begin{array}{l}\text { Keller } \\
\text { See }\end{array}$ & Schöhsee \\
\hline$N$ & 65 & 25 & 20 & 20 & 62 & 25 & 19 & 18 \\
\hline$K$ & 32 & 15 & 12 & 9 & 7 & 5 & 3 & 4 \\
\hline$\theta_{S}$ & 6.32 & 5.03 & 4.51 & 3.10 & 2.98 & 3.44 & 2.00 & 4.07 \\
\hline$\theta_{\pi}$ & 3.28 & 3.40 & 3.88 & 1.63 & 3.81 & 4.44 & 2.00 & 4.51 \\
\hline$\theta_{K}$ & 26.13 & 14.91 & 11.75 & 5.72 & 2.22 & 1.59 & 0.74 & 1.28 \\
\hline Tajima's $D$ & $-1.55^{*}$ & -1.16 & -0.52 & $-1.68 *$ & 0.81 & -0.17 & 0.51 & -0.34 \\
\hline Fu's $F$ & $-12.37 *$ & $-7.14 *$ & -3.84 & $-4.22 *$ & 2.59 & 4.16 & 3.12 & 4.67 \\
\hline
\end{tabular}

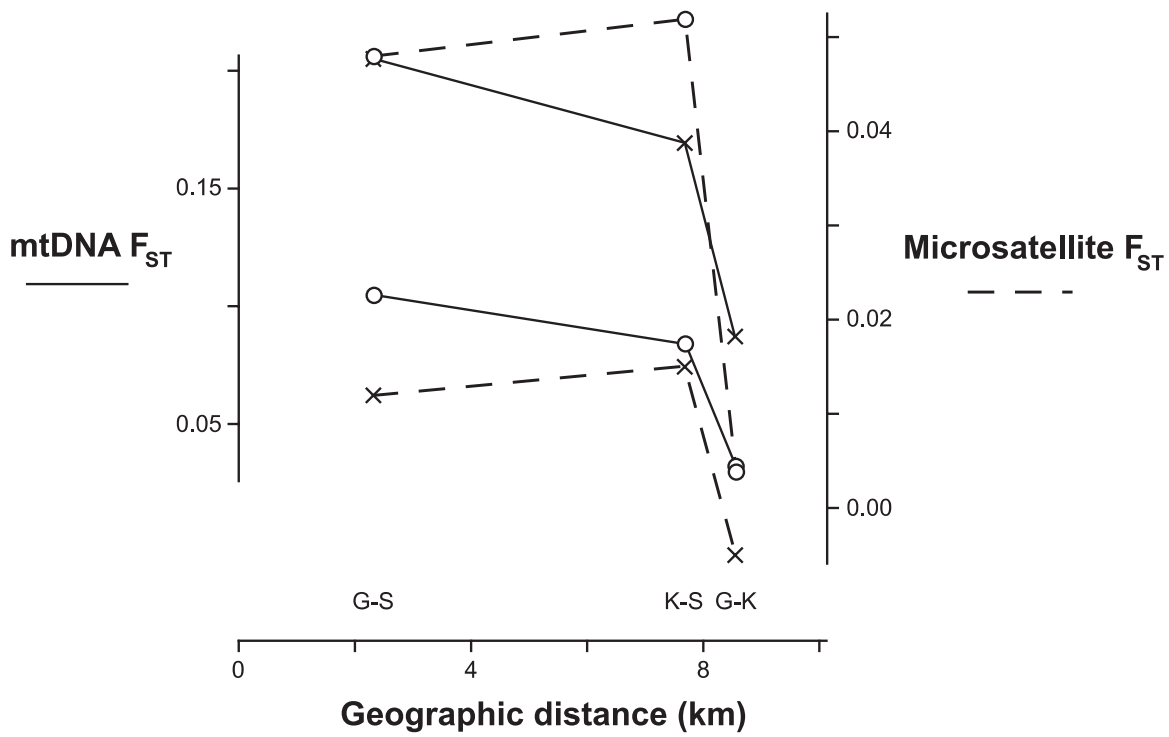

Fig. 1. Estimates of $F_{S T}$ from mtDNA (solid line) and microsatellites (dashed line) between pairs of lakes for E. gracilis (circles) and E. graciloides (crosses). Lake pairs are labeled along the geographic distance axis for the Grosser Plöner See the Schöhsee, and the Keller See.

sites (30 vs. 14) and over four times as many unique haplotypes (32 vs. 7; see Table 1). This led to large discrepancies between $\theta$ estimated from $K$ and $S$, and $\theta$ estimated from $\pi$ (Table 1 ). As a result, Fu's $F$ and Tajima's $D$ showed significant departures from neutral evolution in E. gracilis $(\mathrm{p}<0.05)$, whereas neither of these tests was statistically significant in E. graciloides. Furthermore, the null hypothesis of a sudden population expansion was accepted in 
Table 2. Genetic differentiation among lakes for each species. $* \mathrm{p}<0.05, * * \mathrm{p}<0.01$, $* * * \mathrm{p}<0.001, * * * * \mathrm{p}<0.0001, * * * * * \mathrm{p}<0.00001,{ }^{1}$ significance cannot be determined, although $3 / 3$ loci $>0$.

\begin{tabular}{|c|c|c|c|c|c|c|c|c|}
\hline \multirow[b]{2}{*}{ Contrast } & \multicolumn{4}{|c|}{ E. gracilis } & \multicolumn{4}{|c|}{ E. graciloides } \\
\hline & $\begin{array}{l}\text { across all } \\
\text { lakes }\end{array}$ & G vs. K & G vs. S & $\begin{array}{l}\text { K vs. S } \\
\text { lakes }\end{array}$ & across all & G vs. K & G vs. S & K vs. S \\
\hline \multicolumn{9}{|l|}{ mtDNA } \\
\hline Exact test & $* * * * *$ & $*$ & $* *$ & $* * *$ & $* * * * *$ & & $* * * *$ & $* * * *$ \\
\hline$F_{S T}$ & $0.07 * *$ & 0.03 & $0.11 * *$ & $0.08 * *$ & $0.16 * * *$ & 0.09 & $0.20 * * *$ & $0.17 * *$ \\
\hline$\Phi_{S T}$ & $0.12 * * *$ & $0.07 *$ & $0.09 * *$ & $0.22 * * * * *$ & 0.04 & 0.07 & -0.001 & 0.05 \\
\hline \multicolumn{9}{|c|}{ microsatellites } \\
\hline$F_{S T}$ & $0.035^{*}$ & 0.004 & $0.048 *$ & $0.052 * *$ & $0.009^{1}$ & -0.005 & $0.012^{1}$ & $0.015^{1}$ \\
\hline
\end{tabular}
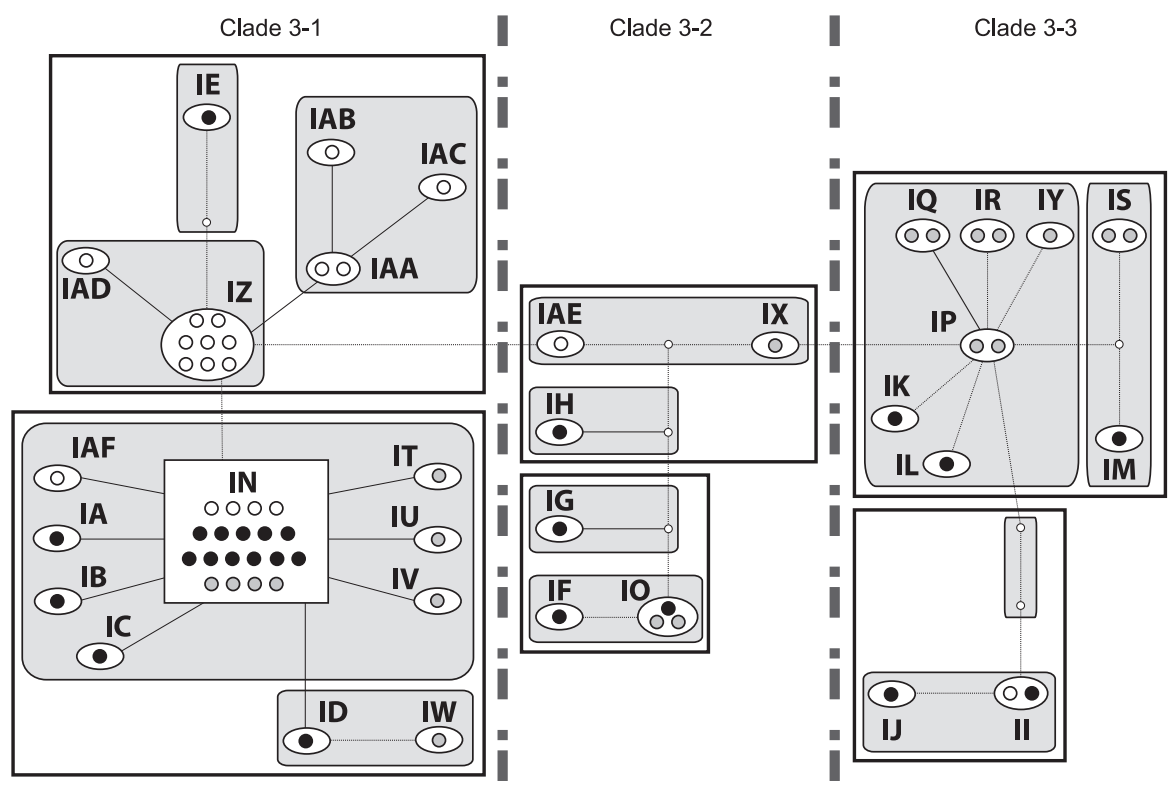

\section{O Schöhsee \\ - Grosser PIöner See O Keller See}

Fig. 2. Haplotype network for E. gracilis (without diapause). Each haplotype is represented as an oval and labeled with a two letter code. Size is proportional to the number of individuals that possess the haplotype. Each line is one mutation, and empty circles represent hypothetical intermediate haplotypes that were not sampled. Boxes and dashed lines separate clades at three hierarchical levels. Haplotype IN (square) has the highest probability of being ancestral. Two ambiguous "loops" (i. e., incompletely resolved portions of the network) were resolved using the criteria of CRANDALL, Templeton and colleagues (CRANDAll et al. 1994, Templeton et al. 1995). 


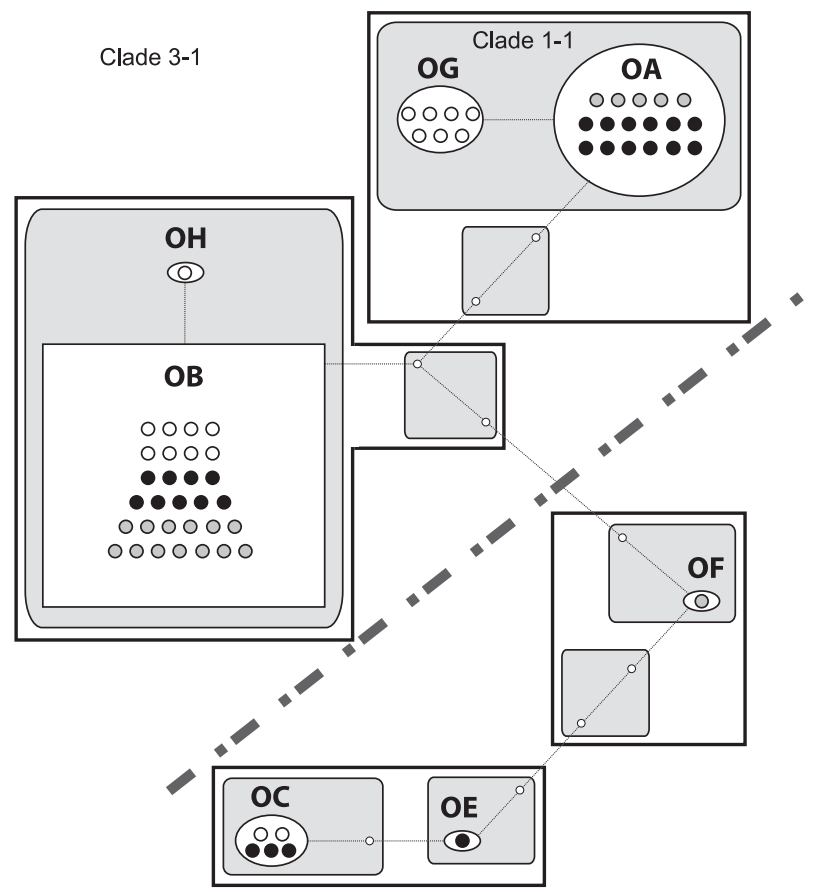

O Schöhsee

- Grosser PIöner See

O Keller See

Fig. 3. Haplotype network for E. graciloides (with diapause). Haplotype OB is hypothesized to be ancestral.

E. gracilis and rejected in E. graciloides (p $<0.05$ for all three lakes and for the pooled data set). Consistent with the mtDNA data, microsatellite variation for the three lakes was higher in E. gracilis $\left(\bar{H}_{e}=0.85-0.87\right.$ in each lake) than in E. graciloides $\left(\bar{H}_{e}=0.78-0.83\right)$.

For mtDNA, exact tests of allele distributions and $F$-statistics both demonstrate that the two copepods have diverged genetically among the lakes. Relative levels of differentiation from microsatellites (estimated as $F_{S T}$ ) and mtDNA $\left(\Phi_{S T}\right.$ and $F_{S T}$ ) were generally consistent with each other, despite some interesting discrepancies (Fig. 1, Table 2). For mtDNA, $\Phi_{S T}$ was higher than $F_{S T}$ in $E$. gracilis, while the opposite was true in E. graciloides. Mitochondrial $F_{S T}$ and microsatellite $F_{S T}$ were both higher in E. gracilis than in E. graciloides, but mtDNA $F_{S T}$ was higher in $E$. graciloides. Lake by lake comparisons also showed differences between some estimates of divergence and hydrologic connectivity. For example, the Keller See and the Grosser Plöner See are sepa- 
rated by the greatest geographic distance, but possess hydrologic connections hospitable to copepods. As a result, these two lakes should have the lowest pairwise genetic divergence, which was true for $F_{S T}$ in both species for both types of markers, and for mtDNA $\Phi_{S T}$ in E. gracilis. Conversely, the Schöhsee and the Keller See should possess the lowest levels of connectivity (since both are upstream of the Grosser Plöner See in different directions). The Schöhsee and the Keller See were the most divergent lakes in E. gracilis for microsatellites and for $\Phi_{S T}$ from mtDNA, but only for microsatellites in $E$. graciloides (Table 2).

Haplotype networks demonstrate the dramatic genetic differences between these species both within and among populations. E. gracilis possesses two common alleles and 29 with frequencies of 1-3\%. Only three alleles are found in more than one lake. E. graciloides also had three alleles present in more than one lake, but had only three common alleles and four rare alleles in total. Geographic patterns of population subdivision were obvious in both species (Figs 2, 3), although the higher levels of genetic diversity in E. gracilis facilitate more inferences about evolutionary processes. In E. gracilis, significant (p $<0.05$ ) geographic associations were found in Clades 3-1, 3-3 and for the total cladogram (see Fig. 2). NCPA suggested contiguous range expansions for clades 3-1 and 3-3, and either a range expansion or restricted gene flow at the level of the total cladogram. For E. graciloides, NCPA found significant geographic associations $(\mathrm{p}<0.0001)$ and evidence for recent allopatric fragmentation in Clade 1-1, representing isolation of the Schöhsee from the other lakes. The older Clade 3-1 shows evidence for restricted gene flow with isolation by distance across all three lakes (Fig. 3), although the tests of geographic association are only marginally significant ( $\mathrm{p}$-values in six associated tests range from 0.07 to 0.09 ).

The Bayesian consensus genealogy for E. graciloides was completely congruent with the parsimony network in Fig. 3. The Bayesian consensus for E. gracilis was nearly congruent with Fig. 2, conflicting only because haplotypes IG and IH were monophyletic in the Bayesian tree (weakly supported with a posterior probability of $55 \%$ ). As a result, the Bayesian genealogies are not presented here. Topologies in the Bayesian genealogies were interpreted in a skyline plot that depicts mutation-scaled effective population size over time (Fig. 4). E. gracilis appears to have an effective population size an order of magnitude higher than E. graciloides, although the contrast is statistically significant only for a brief point during their history. Both species have experienced range and/or population expansions, but the time scales are markedly different. The range expansions inferred from NCPA and the neutrality tests in E. gracilis predate (in generations) a more recent increase in effective population size for E. graciloides. However, E. graciloides has a longer generation time due to the diapausing egg bank in this species. To compare the absolute 


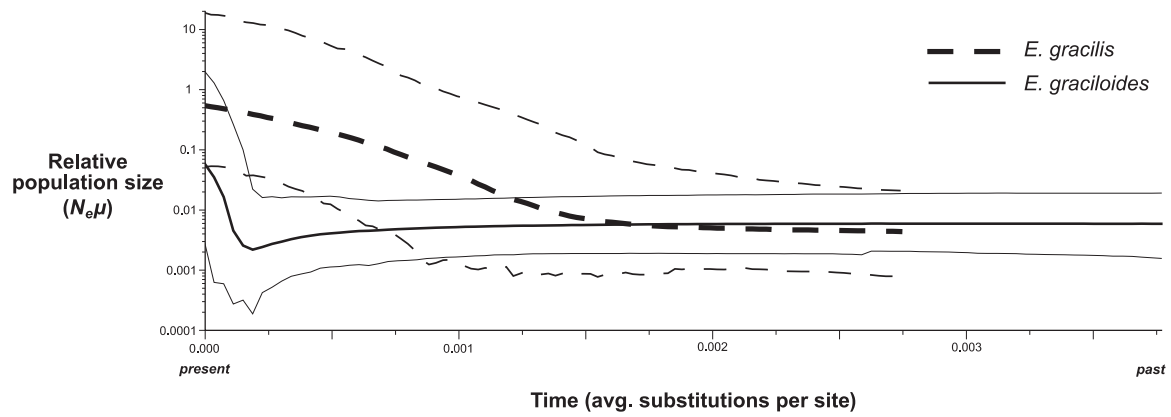

Fig. 4. "Skyline plot" of $N_{e} \mu$ backwards in time (average number of substitutions per site) for E. gracilis (without diapause, dashed lines) and E. graciloides (with diapause, solid lines). The median value and a $95 \%$ confidence interval are shown for each species.

timing of these events, we estimated generation times for both species as follows.

Because E. gracilis has nonoverlapping generations and 2 or 3 generations per year (Hofmann 1979, SANTER et al. 2000, SANTER, unpubl.), we assumed a mean generation time of 0.4 years. For organisms with dormant propagule banks, NunNEY (2002) defined generation time as the average age of all parents, regardless of whether they hatch from subitaneous (immediately hatching) eggs or the diapausing egg bank. Thus, mean generation time for E. graciloides will be the average for animals derived from subitaneous eggs (weighted by the water-column population size), and animals hatching from the egg bank (weighted by the number of diapausing eggs in the lake sediments and the fraction of these that hatch each year). Like E. gracilis, E. graciloides also has a generation time of 0.4 years for eggs that immediately hatch in the water column (Hofmann 1979, SANTER et al. 2000, Santer, unpubl.). Based on lake volumes calculated by SANTER et al. (2000) and densities reported by HofMANN (1979), the water column population sizes are estimated to be $5.10 \times 10^{10}, 6.45 \times 10^{10}$ and $2.15 \times 10^{12}$ in the Schöhsee, the Keller See and the Grosser Plöner See, respectively. Hofmann (1979) showed for the period of his study that population sizes remain fairly constant throughout 2-3 water column generations, suggesting moderate to strong density regulation.

Because we do not have complete information on egg bank dynamics in $E$. graciloides, we rely here on data from another diaptomid copepod population. Diapausing eggs hatch from the well mixed zone in the top $2 \mathrm{~cm}$ of lake sediments (Kearns et al. 1996, Hairston \& Kearns 2002). In the Schöhsee, the recent sedimentation rate is $0.8 \mathrm{~mm}$ per year (HoFMANN 1983). Using this sedimentation rate, the mean age of eggs emerging from the top $2 \mathrm{~cm}$ is 12.5 years. Based on data from SANTER et al. (2000), there are $8.20 \times 10^{9}$ diapausing eggs in the top $2 \mathrm{~cm}$ of the Schöhsee. The annual emergence rate is un- 
known for E. graciloides, so we estimated the proportion hatching from diapause as somewhere between $1 \%$ and $10 \%$ based on data for Onychodiaptomus sanguineus (De Stasio 1989, HaIrston et al. 1995, HaIrston \& KeARns 2002). Taking the $10 \%$ value, the average generation time of the $8.20 \times 10^{8}$ eggs emerging annually from diapause (age 12.5 years) and the $1.28 \times 10^{11}$ subitaneous eggs produced annually in the water column (generation time 0.4 years) is 0.48 years. Comparable calculations for E. graciloides in the Keller See and the Grosser Plöner See produced estimates of 0.81 years and 0.41 years per generation, respectively. Averaging over all the three lakes, we estimate 0.57 years per generation. Thus, the E. graciloides egg bank increases generation time by 0.17 years $(41 \%)$ in comparison with $E$. gracilis. The difference in generation times does not adequately explain the extreme time difference between the two species for start of the population growth phase. Depending on whether the upper or lower confidence intervals in Fig. 4 are used for calculation, the growth phase in E. gracilis begins 5.5-13.7 times earlier. Because generation time in E. graciloides is only $41 \%$ longer than in E. gracilis, the diapausing egg bank of $E$. graciloides cannot account entirely for this discrepancy.

We performed a limited sensitivity analysis on the generation time calculations by arbitrarily changing the fraction of diapausing eggs that emerge annually, since this is the only portion of the calculation that we inferred from another species. Increasing the hatching fraction to $25 \%$ yields an average generation time of 0.59 years, still insufficient to account for differences in timing of the growth phase. Even if $100 \%$ of the eggs in the upper $2 \mathrm{~cm}$ of lake sediment hatched annually, generation time is only increased by a factor of 3.3. Other parameter combinations that would increase generation time by at least 5.5 times are also improbable. For example, the timing of population growth in Fig. 4 could be the same in both species if water column populations of E. graciloides are $88 \%$ smaller than estimated, or if adults hatching from diapausing eggs are on average 374 years old.

In summary, E. graciloides has colonized this region more recently than $E$. gracilis. Regional population size in E. gracilis appears to have increased by two orders of magnitude, leading to an excess of rare, recent mutations. Growth of E. graciloides populations has been less dramatic (approximately one order of magnitude), and follows a decline in effective population size consistent with a bottleneck. It is not possible to date these events precisely without assuming (and calibrating) a molecular clock, and it is possible that generation time has recently decreased in both species due to cultural eutrophication. However, it is clear that the growth phase in each species predates any recent human-induced changes in lake ecology or connectivity, and probably occurred thousands of years ago in E. graciloides, and on the order of ten thousand years ago in E. gracilis. 


\section{Discussion}

Prolonged diapause has the potential to influence patterns of genetic variation within and among populations. However, its attributes may have contradictory effects on factors commonly considered important for the genetic structure of natural populations. Diapausing egg banks should increase population size and generation times, permitting founder effects associated with colonization to persist for thousands of generations or longer (BoILEAU et al. 1992, GómEz et al. 2002). Conversely, diapausing eggs are also assumed to confer high dispersal ability, because the encysted eggs of many species can be passively dispersed in wind or survive gut passage through dispersing vertebrates (e. g., GreEN et al. 2002). This should lead to greater genetic homogenization of populations, and the rapid dissolution of founder effects if gene flow is high enough. Depending on the relative importance of these factors, high genetic differentiation in species with prolonged diapause may reflect either founder effects or low gene flow despite high dispersal; similarly, the absence of genetic differentiation can be attributed either to a homogeneous founder event or to high gene flow. The analysis of neutral DNA sequence data can help distinguish among some alternative interpretations, because time is an inherent part of haplotype networks, with the most recent mutations found at the tips of the genealogy.

For microsatellites, ZeLLER et al. (2006) found patterns of isolation by distance (IBD) in E. graciloides on scales $<100 \mathrm{~km}$, but not in E. gracilis. The absence of IBD in their data for E. gracilis and high variance in population divergence at even the smallest spatial scales could be due either to patterns of gene flow that are irregular and not distance dependent, or to persistent founder effects and very low ongoing gene flow. Phylogeographic and demographic analyses of mtDNA sequence data allow for a more precise interpretation. Historical growth in population sizes are evident in both species (Fig. 4). In $E$. gracilis, this growth phase corresponds to range expansions seen in the oldest (most inclusive) clades. Thus, the mtDNA genealogy reflects the initial colonization of this region by this species (likely just post-Pleistocene). Standard neutrality tests detected a relative excess of unique mtDNA haplotypes in E. gracilis (Table 1), as would be expected for a range expansion combined with an associated growth in effective population size. Because population size increased so dramatically (Fig. 4), founder effects still persist in this species.

Increases in E. graciloides regional population size came at a much later time, possibly associated with a bottleneck in population size (Fig. 4). Founder effects in this species were apparently less pronounced than in E. gracilis, because changes in effective population size have been less dramatic (Fig. 4). This is probably the reason that the null hypothesis of neutral evolution is not 
rejected in $E$. graciloides using simple statistics such as TAJIMA's $D$ and FU's F. Patterns of microsatellite differentiation for E. graciloides suggest that gene flow is highest among lakes that are closer together (ZELLER et al. 2006). However, the mtDNA genealogy shows very low levels of gene flow currently between the Schöhsee and the other two lakes (Clade 1-1 in Fig. 3), as well as unsorted ancestral variation (haplotype OB). Thus, overland gene flow via animal or wind vectors must be very rare, even when populations are separated by $<3 \mathrm{~km}$. We acknowledge the limitations that must accompany any mtDNA population genetic study, including the assumption of selective neutrality and the inherent stochasticity associated with gene trees (KNOWLES \& MADDISON 2002). However, our interpretations are strengthened by an absence of amino acid polymorphisms, and qualitative agreements between the mtDNA and microsatellite loci in terms of intraspecific variation and divergence among populations.

Significant divergence among lakes in mitochondrial and nuclear markers demonstrates that gene flow is low in these copepods, even on small spatial scales among connected lakes. Discrepancies between $\Phi_{S T}$ and $F_{S T}$ estimates also demonstrate that genetic differentiation has developed more rapidly in the absence of diapause. Both of these statistics quantify population differentiation, but $\Phi_{S T}$ accounts for the evolutionary distances between alleles as well as their frequencies. E. gracilis has been isolated for enough generations that lake-specific lineages have evolved through mutation and drift (Fig. 2), so that $\Phi_{S T}>F_{S T}$. In E. graciloides, we interpret $F_{S T}>\Phi_{S T}$ to mean that colonization events involved haphazard assemblages of alleles, and the only new lake-specific mutation to have reached appreciable frequencies is haplotype OG, restricted to the Schöhsee (Fig. 3). The microsatellite data provide additional support for this interpretation. Microsatellite mutation rates are several orders of magnitude higher than those for typical nucleotide sequences. Because $E$. gracilis has apparently been established in the lakes we studied for a longer period of time than E. graciloides, E. gracilis has had more time for unique microsatellite alleles to evolve, and population differentiation is higher. The poor overall match in E. graciloides between levels of divergence between pairs of lakes and hydrologic connectivity provides further evidence that in this species, the lakes we studied are not yet in drift-gene flow equilibrium. Our estimates of generation time suggest that the diapause egg bank of E. graciloides has slowed the decay of genetic founder effects, although not as much as one might initially suspect. It is likely that these results extend to traits under natural selection, although the specific patterns would depend on the strength of selective differences among lakes.

The broad geographic distributions of E. graciloides and E. gracilis (Fig. 5) appear to be consistent with our interpretation of the genetic patterns. Although there has not been a systematic biogeographic study of these two spe- 


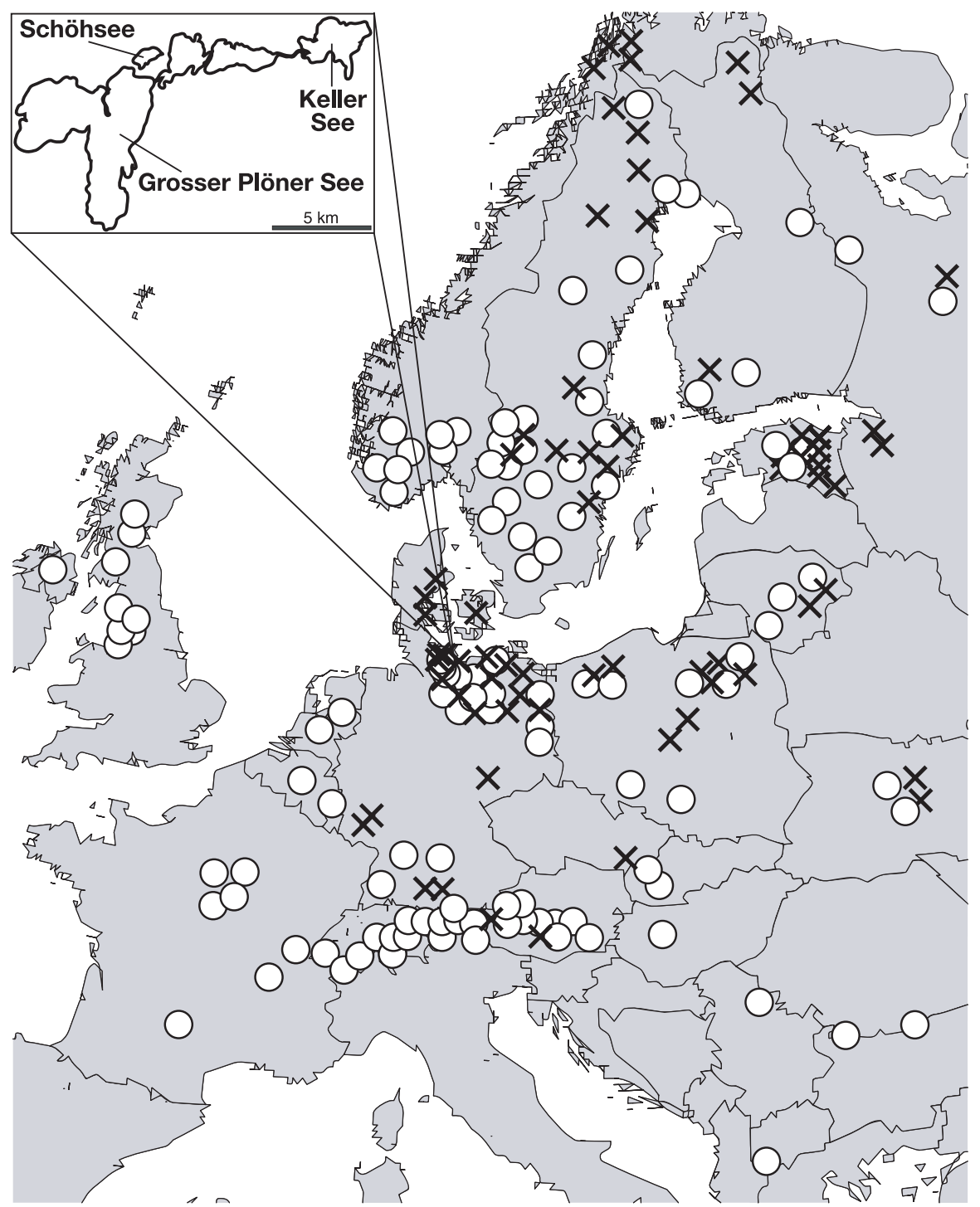

Fig. 5. Occurrence of E. gracilis (circles) and E. graciloides (crosses) in Europe, as reported in previously published studies (references available from the author on request). One symbol may refer to multiple bodies of water in areas where they are closely spaced. KIEFER (1978) also stated that E. gracilis is found as far east as Siberia, and that the range of E. graciloides extends even further to China.

cies, published data from a variety of sources demonstrate that E. gracilis is broadly distributed throughout Europe, and the three lakes included in our study lie near the center of this range. In contrast, our study lakes occur at the 
western edge of the distribution of the E. graciloides, an observation consistent with this species having arrived in our study area more recently.

NAUWERCK (1980) has previously noted that E. gracilis is more common throughout Sweden. However, in northern Sweden, this species is found only near the Baltic Sea shoreline, corresponding to an area that was at times inundated after the last ice age. In contrast, E. graciloides was nearly absent in southern Sweden, more abundant in northern Sweden, and found in mountain regions up to $1000 \mathrm{~m}$. NAUWERCK suggested that E. gracilis was the original inhabitant of continental Europe, and colonized southern Sweden and Britain at a time when lower sea levels provided land bridges (with freshwater bodies) that are not present today. E. graciloides was assumed to have colonized Scandinavia from the east following glacial retreat in this region. NAUWERCK also hypothesized that $E$. gracilis might have inhabited what is now the Baltic Sea during its freshwater phases: the Baltic Ice Lake $>10,000$ years before present, and the Ancylus Lake ca. 8500 years before present. This could have provided the source for E. gracilis to colonize lakes near the northern Sweden shoreline. These hypotheses seem to be generally supported by our analyses, and the large amount of mtDNA diversity in E. gracilis could be explained by a large regional population of E. gracilis in the Baltic Ice Lake and the Ancylus Lake.

Although the presence of diapausing propagule banks is expected to slow drift through increased effective population sizes, we did not find such an effect in E. graciloides. Coalescent analyses suggest that effective population sizes may be an order of magnitude lower in E. graciloides than in E. gracilis, and there is no indication that $E$. gracilis goes through (contemporary) population bottlenecks of any consequence. The smaller effective population size in E. graciloides has led to lower levels of variation for both mtDNA and microsatellites in this species. Independent estimates of effective population size from a temporal analysis of microsatellite variation confirm that population sizes in E. gracilis are comparable to those in E. graciloides, if not larger (ZeLLER et al., unpubl.).

In summary, we sought to assess the genetic effects of prolonged dormancy empirically with as few confounding factors as possible. The copepods E. gracilis and E. graciloides are ecologically and trophically similar, but diapause occurs only in E. graciloides. We focused on lakes where the two species are found sympatrically, and on a spatial scale of $<10 \mathrm{~km}$ to minimize differences among the habitats in terms of regional history and abiotic factors. We found dramatic differences among patterns of genetic differentiation between these two species that we had not predicted based on their life histories. These were at least in part due to differences in their colonization sequence in the region. Interestingly, we found no support for the hypothesis that species with prolonged diapause possess larger effective population sizes than species without diapause. The clear persistence of founder effects in E. graciloides 
precludes an accurate estimate of gene flow in this species. However it is clear that even on small spatial scales, gene flow is not high enough to homogenize populations in either species.

\section{Acknowledgements}

This research began as a challenge from WINFRIED LAMPERT to find diapausing eggs of Eudiaptomus in the Schöhsee: we did and we didn't, and that turned out to be interesting. We thank E. L. Gross, C. A. Cáceres and E. Blohm-Sievers for help in the lab and field, C. A. Aquadro, S. M. Bogdanowicz, C. A. CÁceres, R. G. Harrison, W. LAMPERT, T. Reusch and R. A. Thum for productive discussions. The Bohonak lab provided comments on an early version of the manuscript. Sequencing performed at the Cornell University Evolutionary Genetics Core Facility. The research was funded by a cooperative international travel grant from the U.S. National Science Foundation (INT-9603204) to NGH and the Deutscher Akademischer Austauschdienst to W. LAMPERT, the Max-Planck Society, a Research Experiences for Undergraduates supplement to NSF grant DEB-9815365 to MDH and NGH, and a Cornell University undergraduate research grant to $\mathrm{MDH}$.

\section{References}

Adrian, R. (1997): Calanoid-cyclopoid interactions: Evidence from an 11-year field study in a eutrophic lake. - Freshwat. Biol. 38: 315-325.

Belyea, L. R. \& LANCASTER, J. (1999): Assembly rules within a contingent ecology. Oikos 86: 402-416.

Bilton, D. T., FreEland, J. R. \& OKAMURA, B. (2001): Dispersal in freshwater invertebrates. - Annu. Rev. Ecol. Systemat. 32: 159-181.

BoHonaK, A. J. \& JENKINS, D. G. (2003): Ecological and evolutionary significance of dispersal by freshwater invertebrates. - Ecol. Lett. 6: 782-796.

BohonaK, A. J. \& Roderick, G. K. (2001): Dispersal of invertebrates among temporary ponds: are genetic estimates accurate? - Israel J. Zool. 47: 367-386.

Boileau, M. G., Hebert, P. D. N. \& Schwartz, S. S. (1992): Non-equilibrium gene frequency divergence: persistent founder effects in natural populations. - J. Evol. Biol. 5: 25-39.

BRENDONCK, L., HAMER, M. \& Thiery, A. (1992): Occurrence of tetrahedral eggs in the Streptocephalidae Daday (Branchiopoda: Anostraca) with descriptions of a new subgenus, Parastreptocephalus and a new species, Streptocephalus (Parastreptocephalus) zuluensis Brendonck and Hamer. - J. Crustacean Biol. 12: $282-$ 297.

BRENDONCK, L. \& RidDOCH, B. J. (1999): Wind-borne short-range egg dispersal in anostracans (Crustacea: Branchiopoda). - Biol. J. Linnean Society 67: 87-95.

CÁceres, C. E. \& Soluk, D. A. (2002): Blowing in the wind: a field test of overland dispersal and colonization by aquatic invertebrates. - Oecologia 131: 402-408.

Clement, M., Posada, D. \& Crandall, K. A. (2000): TCS: a computer program to estimate gene genealogies. - Molecular Ecol. 9: 1657-1659. v. 1.16. 
Cohen, G. M. \& Shurin, J. B. (2003): Scale-dependence and mechanisms of dispersal in freshwater zooplankton. - Oikos 103: 603-617.

Cousyn, C., De Meester, L., Colbourne, J. K., Brendonck, L., Verschuren, D. \& VolCKAERT, F. A. M. (2001): Rapid, local adaptation of zooplankton behavior to changes in predation pressure in the absence of neutral genetic changes. - Proc. National Acad. Sci. United States of America 98: 6256-6260.

Crandall, K. A., Templeton, A. R. \& Sing, C. F. (1994): Intraspecific phylogenetics: Problems and solutions. - In: Scotland, R. W., Siebert, D. J. \& Williams, D. M. (eds): Models in phylogeny reconstruction. - Oxford, Clerendon, pp. 273 297.

De Meester, L., Gómez, A., Okamura, B. \& Schwenk, K. (2002): The Monopolization Hypothesis and the dispersal-gene flow paradox in aquatic organisms. - Acta Oecologica 23: 121-135.

De Stasio, B. T. Jr. (1989): The seed bank of a freshwater crustacean: copepodology for the plant ecologist. - Ecology 70: 1377-1389.

Drummond, A. J. \& Rambaut, A. (2003): BEAST (Bayesian Evolutionary Analysis Sampling Trees), 1.2. http://evolve.zoo.ox.ac.uk/beast/

Drummond, A. J., Nicholls, G. K., Rodrigo, A. G. \& Solomon, W. (2002): Estimating mutation parameters, population history and genealogy simultaneously from temporally spaced sequence data. - Genetics 161: 1307-1320.

Drummond, A. J., Rambaut, A., Shapiro, B. \& Pybus, O. G. (2005): Bayesian coalescent inference of past population dynamics from molecular sequences. - Molecular Biol. Evol. 22: 1185-1192.

EllNER, S. P. \& HAIRSTON, N. G. Jr. (1994): Role of overlapping generations in maintaining genetic variation in a fluctuating environment. - Amer. Nat. 143: 403-417.

Ellner, S. P., Hairston, N. G. Jr., Kearns, C. M. \& BabAi, D. (1999): The roles of fluctuating selection and long-term diapause in microevolution of diapause timing in a freshwater copepod. - Evolution 53: 111-122.

Excoffier, L., Smouse, P. E. \& Quattro, J. M. (1992): Analysis of molecular variance inferred from metric distances among DNA haplotypes: application to human mitochondrial DNA restriction data. - Genetics 131: 479-491.

FU, Y.-X. (1997): Statistical tests of neutrality of mutations against population growth, hitchhiking and background selection. - Genetics 147: 915-25.

Gómez, A., Adcock, G. J., Lunt, D. H. \& CARvalho, G. R. (2002): The interplay between colonization history and gene flow in passively dispersing zooplankton: microsatellite analysis of rotifer resting egg banks. - J. Evol. Biol. 15: 158-171.

GoudET, J. (1995): FSTAT: A computer program to calculate F- statistics. - J. Heredity 86: 485 -486. v. 2.9.3.2.

Green, A. J., Figuerola, J. \& Sanchez, M. I. (2002): Implications of waterbird ecology for the dispersal of aquatic organisms. - Acta Oecologica 23: 177-189.

HAIRston, N. G. Jr. (1996): Zooplankton egg banks as biotic reservoirs in changing environments. - Limnol. Oceanogr. 41: 1087-1092.

Hairston, N. G. Jr. \& De Stasio, B. T. Jr. (1988): Rate of evolution slowed by a dormant propagule pool. - Nature 336: 239-242.

Hairston, N. G., Jr. \& KeARns, C. M. (2002): Temporal dispersal: ecological and evolutionary aspects of zooplankton egg banks and the role of sediment mixing. Integrative and Comparative Biol. 42: 481-491. 
Hairston, N. G. Jr., Van Brunt, R. A., Kearns, C. M. \& Engstrom, D. R. (1995): Age and survivorship of diapausing eggs in a sediment egg bank. - Ecology 76: 1706-1711.

Halliburton, R. (2004): Introduction to population genetics. - Pearson/Prentice Hall, Upper Saddle River, NJ, pp. 1-650.

HARrison, S. \& Bruna, E. (1999): Habitat fragmentation and large-scale conservation: What do we know for sure? - Ecography 22: 225-232.

HeDrick, P. W. (1995): Genetic polymorphism in a temporally varying environment: effects of delayed germination or diapause. - Heredity 75: 164-170.

Hofmann, W. (1979): Characteristics of syntopic populations of Eudidaptomus gracilis (SARS) and E. graciloides (LILLJEBORG) in three lakes of different trophic levels. - Arch. Hydrobiol. 86: 1-12.

- (1983): Stratigraphy of Cladocera and Chironomidae in a core from a shallow north German lake. - Hydrobiologia 103: 235-239.

Hutchinson, D. W. \& Templeton, A. R. (1999): Correlation of pairwise genetic and geographic distance measures: inferring the relative influences of gene flow and drift on the distribution of genetic variability. - Evolution 53: 1898-1914.

JENKINS, D. G. \& UnDERwoOD, M. O. (1998): Zooplankton may not disperse readily in wind, rain, or waterfowl. - Hydrobiologia 387-388: 15-21.

Kawecki, T. J. \& Ebert, D. (2004): Conceptual issues in local adaptation. - Ecol. Lett. 7: $1225-1241$.

Kearns, C. M., Hairston, N. G. Jr. \& Kesler, D. H. (1996): Particle transport by benthic invertebrates: its role in egg bank dynamics. - Hydrobiologia 332: 63-70.

Kiefer, F. (1978): Freilebende Copepoda. - In: Kiefer, F. \& Fryer, G.: Das Zooplankton der Binnengewässer, 2. Teil. Die Binnengewässer 26. - E. Schweizerbart'sche Verlagsbuchhandlung, Stuttgart, pp. 1-343.

Knowles, L. L. \& Maddison, W. P. (2002): Statistical phylogeography. - Molecular Ecol. 11: 2623-2635.

Korovchinsky, N. M. \& Boikova, O. S. (1996): The resting eggs of the Ctenopoda (Crustacea: Branchiopoda): a review. - Hydrobiologia 320: 131-140.

Louette, G. \& De Meester, L. (2005): High dispersal capacity of cladoceran zooplankton in newly founded communities. - Ecology 86: 353-359.

Nauwerck, A. (1980): The distribution of the Diaptomidae Sars in Northern Sweden. - Arch. Hydrobiol. 89: 247-264.

NunNey, L. (2002): The effective size of annual plant populations: The interaction of a seed bank with fluctuating population size in maintaining genetic variation. Amer. Nat. 160: 195-204.

Posada, D. \& Crandall, K. A. (2001): Intraspecific gene genealogies: trees grafting into networks. - Trends Ecol. Evol. 16: 37-45.

Posada, D., Crandall, K. A. \& Templeton, A. R. (2000): GeoDis: A program for the cladistic nested analysis of the geographical distribution of genetic haplotypes. - Molecular Ecol. 9: 487-488.

Raymond, M. \& Rousset, F. (1995): GENEPOP (Version 1.2): Population genetics software for exact tests and ecumenicism. - J. Heredity 86: 248-249.

Rogers, A. R. \& Harpending, H. C. (1992): Population growth makes waves in the distribution of pairwise genetic differences. - Molecular Biol. Evol. 9: 552-569. 
Santer, B., Blohm-Sievers, E., Caceres, C. E. \& Hairston, N. G. Jr. (2000): Lifehistory variation in the coexisting freshwater copepods Eudiaptomus gracilis and Eudiaptomus graciloides. - Arch. Hydrobiol. 149: 441-458.

Schneider, S., Roessli, D. \& Excoffier, L. (2001): Arlequin: a software for population genetics data analysis, 2.0 (2001 update). http://anthro.unige.ch/arlequin

Simon, C., Frati, F., Beckenbach, A., Crespi, B., Liu, H. \& Flook, P. (1994): Evolution, weighting, and phylogenetic utility of mitochondrial gene sequences and a compilation of conserved polymerase chain reaction primers. - Ann. Entomol. Soc. Amer. 87: 651-701.

SLATKIN, M. (1993): Isolation by distance in equilibrium and non-equilibrium populations. - Evolution 47: 264-279.

TAJima, F. (1989): The effect of change in population size on DNA polymorphism. Genetics 123: 597-601.

Templeton, A. R. (1998): Nested clade analyses of phylogeographic data: testing hypotheses about gene flow and population history. - Molecular Ecol. 7: 413-418.

- (2004): Statistical phylogeography: methods of evaluating and minimizing inference errors. - Molecular Ecol. 13: 789-809.

Templeton, A. R., Boerwinkle, E. \& Sing, C. F. (1987): A cladistic analysis of phenotypic associations with haplotypes inferred from restriction endonuclease mapping: I. Basic theory and an analysis of alcohol dehydrogenase activity in Drosophila. - Genetics 117: 343-352.

Templeton, A. R., Crandall, K. A. \& Sing, C. F. (1992): A cladistic analysis of phenotypic associations with haplotypes inferred from restriction endonuclease mapping and DNA sequence data: III. Cladogram estimation. - Genetics 132: 619-633.

Templeton, A. R., Routman, E. J. \& Phillips, C. A. (1995): Separating population structure from population history: A cladistic analysis of the geographical distribution of mitochondrial DNA haplotypes in the tiger salamander, Ambystoma tigrinum. - Genetics 140: 767-782.

VanoverbeKe, J. \& De Meester, L. (1997): Among-populational genetic differentiation in the cyclical parthenogen Daphnia magna (Crustacea, Anomopoda) and its relation to geographic distance and clonal diversity. - Hydrobiologia 360: 135142.

Walsh, P. S., Metzger, D. A. \& Higuchi, R. G. (1991): Chelex 100 as a medium for simple extraction of DNA for PCR-based typing from forensic material. - Biotechniques 10: 506-513.

WeIDER, L. J. \& HEBERT, P. D. N. (1987): Microgeographic genetic heterogeneity of melanic Daphnia pulex at a low-Arctic site. - Heredity 58: 391-400.

Wright, S. (1931): Evolution in Mendelian populations. - Genetics 16: 97-159.

Zeller, M., Reusch, T. B. H. \& LAMPert, W. (2006): A comparative population genetic study on calanoid freshwater copepods: Investigation of isolation-by-distance in two Eudiaptomus species with a different potential for dispersal. - Limnol. Oceanogr. 51: 117-124.

Submitted: 19 January 2006; accepted: 14 April 2006. 